\title{
Chaos and Complexity in a Simple Model of Production Dynamics
}

\author{
I. KATZORKE and A. PIKOVSKY* \\ Department of Physics, University of Potsdam, Germany
}

(Received 15 March 2000)

\begin{abstract}
We consider complex dynamical behavior in a simple model of production dynamics, based on the Wiendahl's funnel approach. In the case of continuous order flow a model of three parallel funnels reduces to the one-dimensional Bernoulli-type map, and demonstrates strong chaotic properties. The optimization of production costs is possible with the OGY method of chaos control. The dynamics changes drastically in the case of discrete order flow. We discuss different dynamical behaviors, the complexity and the stability of this discrete system.
\end{abstract}

Keywords: Production dynamics; Discrete mapping; Complexity; Control; Quasiperiodicity

\section{INTRODUCTION}

A production system consists of a number of work units, in which sets of different parts are produced corresponding to customer orders. The orders flow through the production system and cause the manufacturing of the requested quantities. As orders arrive at a work system, they form a queue of waiting orders and build up a buffer inventory. The problem of production control can be formulated as that of regulating the flow of orders in such a way that given aims (e.g., minimal costs and due date reliability) are achieved.

The production process is described in a floworiented manner with a funnel model, according to Wiendahl (1987). A funnel represents a work unit, which could be an individual work place, a group of work places, or an operation area. The funnels are connected by the flow of "materials" (parts, orders). From outside, orders are pushed into the system. As they arrive, they fill the funnel and flow out of the funnel after processing. Therefore the funnel opening symbolizes the performance of the work system, which can vary according to the work capacity made available. Irregular oscillation of funnel filling levels may disturb the flow and reduce the performance of the system.

Our approach is to describe the production process as a nonlinear dynamical system (other applications of nonlinear methods to manufacturing systems have been recently pursued by Bunimovich, 1999; Bartholdi et al., 1999; Hanson

${ }^{*}$ Corresponding author. 
et al., 1999; Dias-Rivera et al., 1999). Thus, we consider all outside orders as regular (periodic or even constant) functions of time, and all system parameters as constants. In such circumstances the only source of complex irregular behavior can be the internal nonlinear dynamics. The real production systems are rather complex in the sense of the number of different work places and connections between them. Our aim in this work is to discuss the optimization of the production costs of a simple three-funnel model with continuous flow and chaotic behavior described by Chase et al. (1993); Schürmann and Hoffmann (1995). In particular, we demonstrate that the chaos can be controlled to minimize some cost function. In Section 3 we generalize this simple funnel model by introducing a discrete order flow. The dynamic of the model now depends on the work velocities and can be irregular or periodic. Remarkably, the irregularity is weaker than chaos; in particular the Lyapunov exponent of the mapping is zero. We discuss the complexity properties of this system in details.

\section{A SIMPLE CONTINUOUS FUNNEL MODEL WITH A "STRANGE BILLIARD" AND ITS CONTROL}

The simple funnel model to be considered in this paper consists of one "input" funnel, which is connected with three following ones that work in parallel. At any time the material flow is directed from the first funnel to only one of the three funnels 1, 2, 3 (Fig. 1). To which of these funnels the material flow is directed, depends on the history of the system and is regulated by the following switching rule: if one of the funnels becomes empty, the flow is immediatly switched to this funnel and it remains here until the next one becomes empty. In this section we always assume that the material flow is continuous which means that the switching can occur at any moment of time. We would like to stress that "empty" does not necessarily mean zero level: we can take any level as a reference and measure the contents of each funnel with respect to this level.

If the total production rate of the three funnels is less than the material inflow, the levels of the funnels will grow in average; and if the production rate is larger than the inflow, the levels of the funnels will decrease. Thus, a nontrivial statistically stationary regime is only possible for the balanced system, where the total production rate of the three funnels is equal to the material inflow. Normalizing the input to unity, this means

$$
u_{1}+u_{2}+u_{3}=1
$$

where $u_{i}$ are the production rates of the funnels. From the balance condition it follows, that the

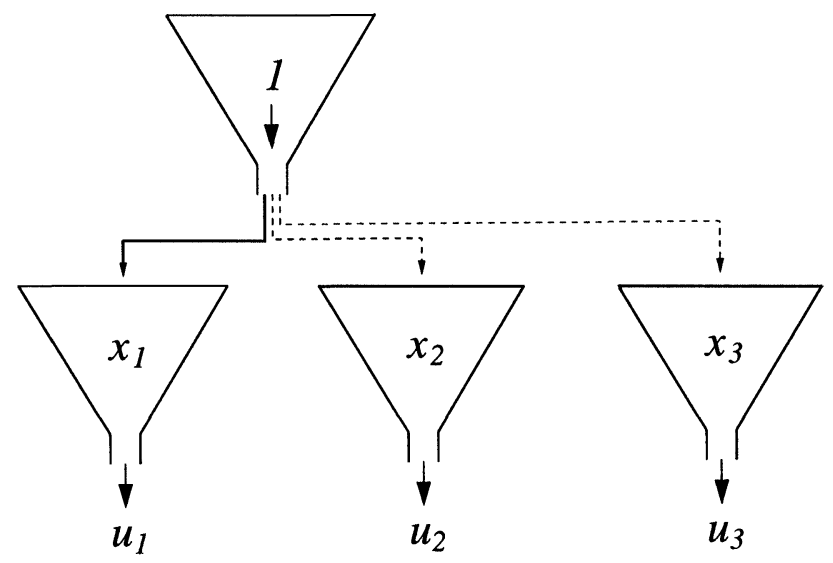

FIGURE 1 A simple funnel model with three funnels. Solid line: funnel $x_{1}$ is filled from the input flow until some other funnel will be empty. Dashed lines illustrate the other two posible cases, when funnel $x_{2}$ or $x_{3}$ is being filled. 
total amount of the material in three funnels is a constant (defined by the initial conditions):

$$
x_{1}+x_{2}+x_{3}=U .
$$

We can write the equations of motion as a set of ordinary differential equations for the contents of the funnels $x_{i}$ :

$$
\dot{x}_{i}=-u_{i}+\delta_{i k},
$$

where the time-dependent index $k$ corresponds to the funnel which is being filled at a given moment of time. An example of the time dependence of $x_{i}$ is shown in Figure 2.

The dynamics of the model is three dimensional, where the coordinates $x_{i}$ (representing the dynamical degrees of freedom) are the contents of the funnels. Because the sum of these filling levels is a constant, a point in the phase space $\left(x_{1}, x_{2}, x_{3}\right)$ which describes the state of the system moves on the plane (2). The condition $x_{i} \geq 0$ defines the triangle which is the phase space of our model. According to (3) the motion is linear until the trajectory hits the boundary of the triangle. At the hit the input flow switches and a new straight line begins. Thus, we have straight line motions with reflections at the boundaries. These dynamics were called "strange billiards" by Schürmann and Hoffmann (1995), because (contrary to the billiards systems usually considered in classical mechanics) the reflection angle is fixed and independent on the incident angle.

As usual in billiard systems, the dynamics can be easily reduced to the mapping of the boundary onto itself - the Poincaré map. This mapping can be obtained by solving the equations of motion (3) between the reflections, the result is a piecewiselinear function having 6 segments of constant slope (Fig. 3). The mapping has one discontinuity point and the absolute value of its slope is larger than one. Therefore it is topologically equivalent to the dyadic Bernoulli map.

Because the Bernoulli map has a natural Markov partition, the invariant probability distribution can be found analytically. According to this distribution, shown in Figure 3, we can find different statistical characteristics of the work process, in particular the cost function. Here we assume that every switching of the inflow direction causes a fixed cost $K$. Then the average costs per time unit are

$$
k=K \lim _{T \rightarrow \infty} \frac{N_{T}}{T},
$$

where $N_{T}$ is the number of switches during the time interval $T$. As we have the statistical description formulated in terms of the Poincaré map, it is easy to find the average time between

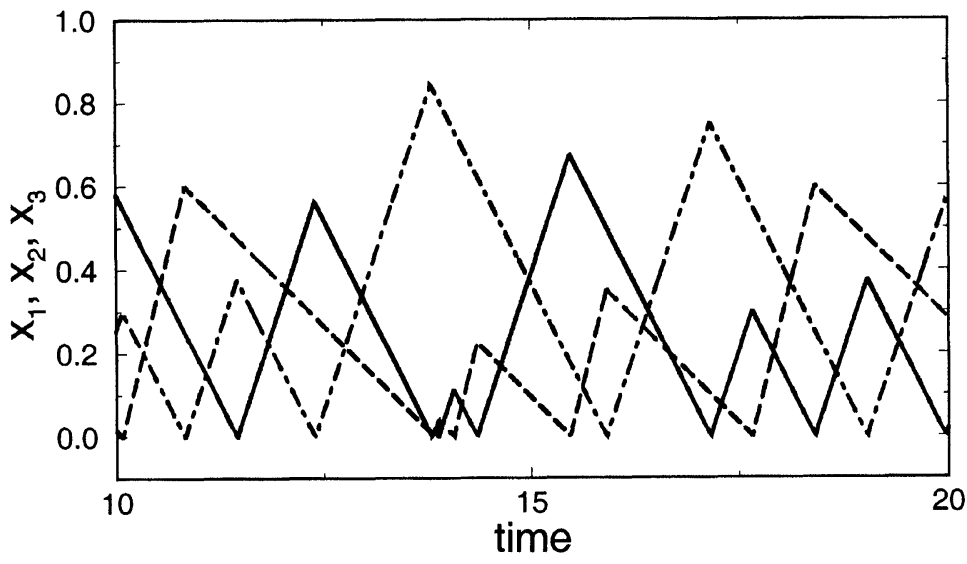

FIGURE 2 The funnel fillings levels $x_{1}$ (solid line), $x_{2}$ (dot-dashed line) and $x_{3}$ (dashed line) as functions of time. The sum of these values is constant, and $x_{1}, x_{2}, x_{3} \geq 0$. The flow rates are $u_{1}=u_{2}=0.4, u_{3}=0.2$, correspondingly the slopes of the curves are different. 

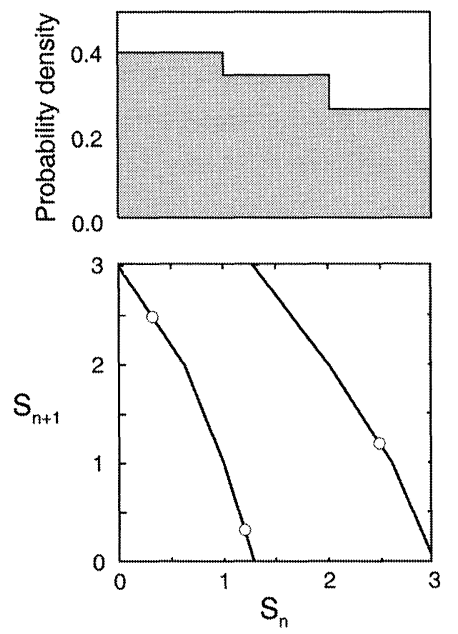

FIGURE 3 Lower panel: The Poincaré map for the threefunnel model with the production rates $u_{1}=0.5, u_{2}=0.3$ and $u_{3}=0.2 . S_{n}$ and $S_{n+1}$ are the successive reflection points of the triangle circumference coordinate $S$. The small circles represent the period-3 orbit that minimizes the cost function. Upper panel: the invariant probability distribution.

switches

$$
\tau=\lim _{N \rightarrow \infty} \frac{T}{N_{T}}
$$

which is inversely proportional to the cost function:

$$
k=\frac{K}{\tau} .
$$

The average time can be easily found analytically through the invariant probability distribution.

We now address the problem of minimizing the cost function, by controlling chaos. According to Ott et al. (1990), it is possible to stabilize periodic orbits inside chaotic regions by using the so-called OGY method of chaos control. If these orbits have a larger mean switching time, then the cost function will have a lower value than for the chaotic regime. It is straightforward to find the periodic points of the Bernoulli map numerically, and to calculate the corresponding mean switching times. The results are shown in Figure 4. The minimum of the cost function is reached on the simplest period-3 periodic orbit when all three funnels are filled in a cyclic manner: $1 \rightarrow 2 \rightarrow 3 \rightarrow$

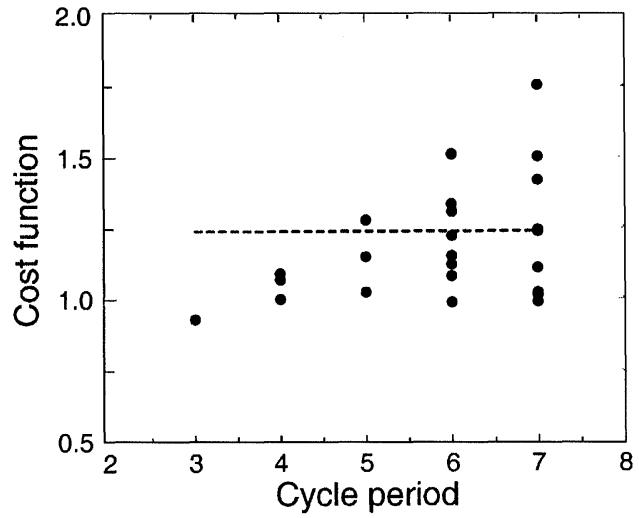

FIGURE 4 The cost function for the cycles of different periods, and the average one (dashed line). The cycle of period 3 yields the global minimum.

$1 \rightarrow 2 \rightarrow 3 \rightarrow 1 \ldots$ Thus, controlling the simplest period -3 orbit minimizes the cost function.

The controlling strategy is straightforward. As one of the funnels is becoming empty, we have to check if the filling levels of the others are smaller or larger than the coordinate of the period-3 orbit. In the former case one has to switch slightly before the funnel is empty, in the latter case one has to switch after the zero level is reached. In this way it is possible to get the trajectory closer to the desired orbit, and finally to stabilize it.

\section{THE SIMPLE FUNNEL MODEL WITH DISCRETE ORDER FLOW}

\subsection{Formulation of the Problem}

In this section we generalize the model discussed above to the case of discrete order flow. We assume that the orders are coming every unit of time. We take the discretization interval to be equal 1 , then the total contents of the funnels is another parameter of the problem. We could also normalize in another way: to assume that the total contents is 1 and the discretization interval is $\Delta t \neq$ 1. Correspondingly, the outflow from the funnels 1-3 happens discretely, at the time intervals $u_{i}^{-1}$.

The equations of motion (3) remain the same, but the switching rule has to be changed. Now the 
switching can occur only at integer times, and we use the following rule: if at time $t=n$ the level of the $i$-th funnel is less or equal to zero Figure 5, then this funnel starts to be filled (it can happen that two funnels have negative levels, in this case we use a prescribed preference scheme). Note that the level of the funnel is determined as a continuous variable in this scheme.

Summarizing, instead of the continuous-time dynamics (3) we have the discrete-time dynamics

$$
x_{i}(t+1)=x_{i}(t)-u_{i}+\delta_{k i}(t) .
$$

The system must be be balanced, i.e., the total input is equal to the total outflow. This property should be formulated for the discrete case as well:

$$
\sum_{i=1}^{3} u_{i}=\sum_{i=1}^{3}\left(\frac{1}{T_{i}}\right)=1
$$

Here $u_{i}$ are the production rates and $T_{i}$ are the times of the work process for one workpiece. From (4) and (5) it follows that the sum of the variables $x_{i}$ is an integral of motion:

$$
\sum_{i=1}^{3} x_{i}=X=\text { const. }
$$

As already mentioned, the value of $X$ is one of the important parameters of the problem.

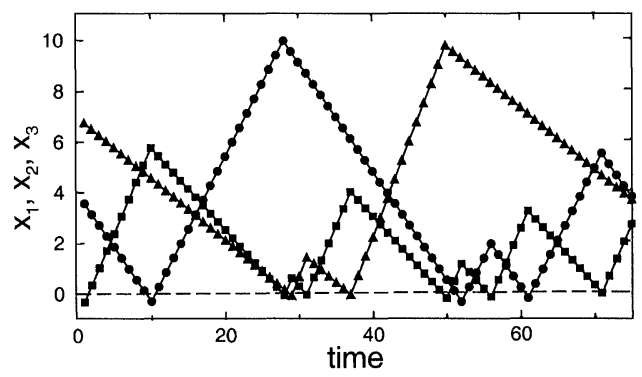

FIGURE 5 The funnel filling levels $x_{1}, x_{2}$ and $x_{3}$ as a function of time. The sum of these values is constant. The markers denote the discrete time. Note that the switching takes place if one of the variables $x_{i}$ becomes negative or zero.

\subsection{Rational Production Rates}

We start with the case when all production rates are rationals

$$
u_{i}=\frac{p_{i}}{q}
$$

with some integers $p_{i}, q$. We show now that all motions in this case are periodic. Let us consider the $q$-th iteration of (4):

$$
x_{i}(t+q)=x_{i}(t)+p_{i}+N_{i}
$$

where

$$
N_{i}=\sum_{\tau=t}^{t+k-1} \delta_{i k}(\tau)
$$

is the total inflow of the funnel $i$ during this time interval. Because $p_{i}$ and $N_{i}$ are integers, it follows from (8) that the fractional parts of $x_{i}$ are integrals of motion. Thus, we have a mapping defined on a bounded set of integers. Therefore, eventually every trajectory is periodic.

This conclusion is supported by numerical experiments, where we for every initial condition determined the period of the emerging periodic orbit. We show the results for three values of the total filling level $X$ in Figure 6. The period in these pictures denotes the total number of switches on the periodic trajectory, the minimal period is obviously 3 . We observe the following features, which appear to be rather unusual for dynamical systems:

1. The dynamics is multistable, and the level of multistability increases with the parameter $X$. Cycles of different period coexist.

2. All the cycles are neutrally stable, moreover, they appear in two-parametric families that fill whole regions on the plane of variables. These regions are very ordered if $X$ is an integer, and less ordered for irrational $X$.

Analytically, the property 2 is a direct consequence of (8): adding small perturbations to $x_{i}$ 
(a)

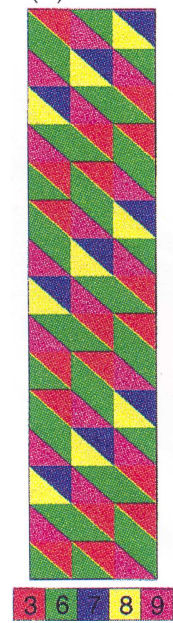

(b)

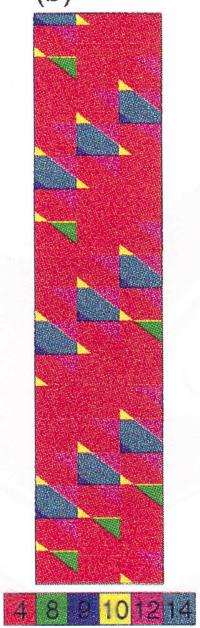

(c)

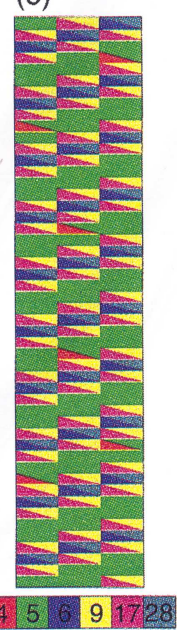

FIGURE 6 The period of the eventual periodic trajectory in dependence on initial point, shown in the initial coordinates $\left(x_{1}, x_{2}\right)$. The production rates are $u_{1}=0.4, u_{2}=0.4, u_{3}=0.2$. The total filling level is $X=3$ in (a), $X=2 \sqrt{3}$ in (b) and $X=10$ in (c). The color/grey codes for the periods are different on different panels. (See Color Plate I.)

does not change the dynamics that is essentially determined by the integer part of the variables $x_{i}$. This property can be also demonstrated geometrically. Consider a closed trajectory as shown in Figure 7. Shifting this trajectory parallel to any of its sides (or a combination of such shifts) leads to another allowed trajectory, if the "nodes" do not cross the borders of the triangle $x_{1}+x_{2}+x_{3}=X$. One can see, that the possible switching points for a given periodic orbit form a triangle, whose corners are given by the condition that two nodes lie exactly on the border of the triangle $x_{1}+x_{2}+x_{3}=X$.

\subsection{Irrational Production Rates}

If at least two production rates $u_{i}$ are irrational, periodic orbits do not exist. This follows simply from the observation, that for irrational $u_{i}$ the condition $x_{i}(t+N)=x(t)$ cannot be fulfilled. $\mathrm{Nu}-$ merical simulations demonstrate that the processes in the system are rather irregular. The situation here is similar to that in the linear parabolic maps, described recently by Zyczkowski and Nishikawa

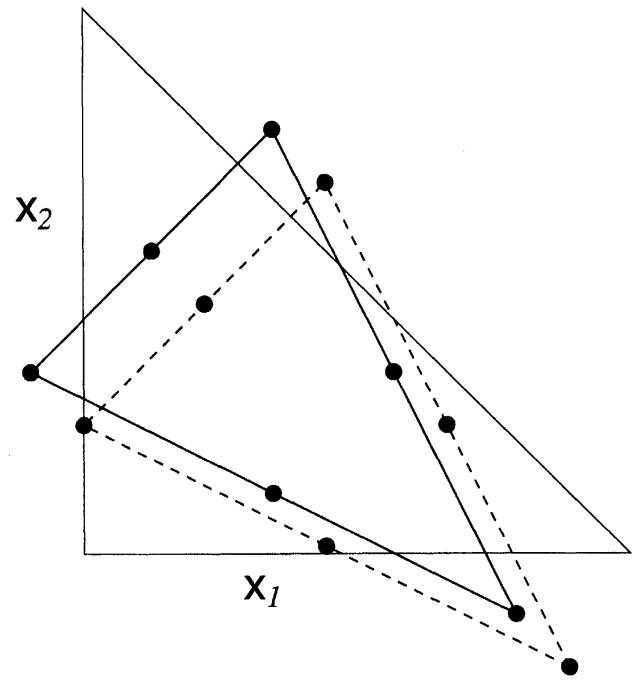

FIGURE 7 A periodic trajectory of the discrete system, shown in $x_{1}-x_{2}$ coordinates (solid line). The dots mark the integer moments of time. The trajectory can be shifted unless the dots do not cross the borders of the triangle; one possible position is shown with the dashed line.

(1999). In all numerical calculations presented below we use the production rates

$u_{1}=\frac{\theta^{2}}{1+\theta+\theta^{2}}, \quad u_{2}=\frac{\theta}{1+\theta+\theta^{2}}, \quad u_{3}=\frac{1}{1+\theta+\theta^{2}}$,

where $\theta=1.3247 \ldots$ is the spiral-mean number (the real root of $\left.\theta^{3}-\theta-1=0\right)$. Also, we consider the fixed total level of the funnels to $X=10$. We have performed numerical experiments with some other parameter values, they demonstrate the same qualitative features.

\subsubsection{Phase Space Portrait}

The mapping defined by (4) is volume-preserved, but not one-to-one. Due to the overlapping of the iterations, the final attractor looks like a set of closed domains, see Figure 8. A similar structure of the attracting set have been observed by Zyczkowski and Nishikawa (1999).

\subsubsection{Complexity}

There are different complexity measures that allows one to characterize a time sequence (see 

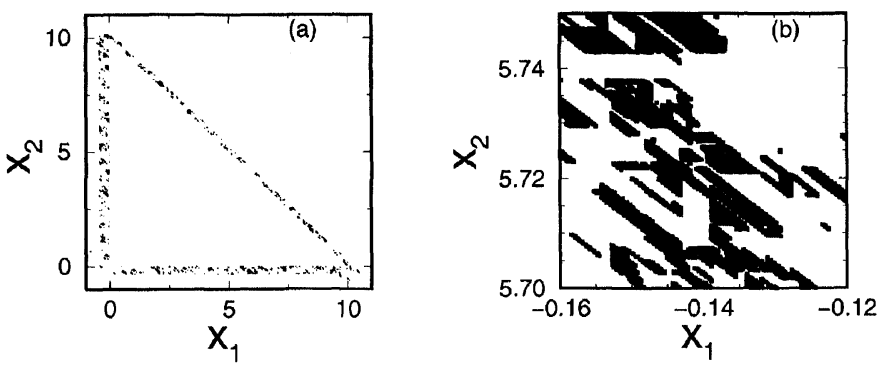

FIGURE 8 The structure of the phase space of the mapping (4). In (a) only points outside the triangle (i.e., the points where switchings occur) are shown. The attractor is built by 10000 points of one trajectory (with transients discarded). A small part of (a) is enlarged in (b); here one can see that the attractor consists of open domains.

Badii and Politi, 1997). The first step is to introduce a symbolic representation of the process. The natural symbolic coding with three symbols $1,2,3$ corresponds to the sequence of the funnels that are filled. Not all combinations of three symbols are possible: a repetition of a symbol is forbidden. This allows us to reduce the symbolic representation to two symbols. Namely, we associate to the transitions $1 \rightarrow 2,2 \rightarrow 3,3 \rightarrow 1$ the symbol 1 , and to the transitions $2 \rightarrow 1,1 \rightarrow 3,3 \rightarrow 2$ the symbol -1 . Then, as one can easy see, the initial state and the sequence of $1 \mathrm{~s}$ and $-1 \mathrm{~s}$ are sufficient to reproduce the full sequence of three symbols 1,2,3. Remarkably, in the new two-symbol representation all combinations of two symbols are possible. So the maximal possible number of different words of length $n$ is $2^{n}$. This number will be realized if the sequence is random. For chaotically generated sequences (e.g., this is the case for the continuousflow system described in Section 2 above) one expects the number $N_{n}$ of different words of length $n$ to grow exponentially $N_{n} \propto e^{h n}$, where $h$ is the topological entropy of the system (Katok and Hasselblatt, 1995).

To characterize the complexity, we have found all sequences up to length 30 , the results are presented in Figure 9. The number $N_{n}$ increases definitely slower than any exponent, the best fit for $n=13, \ldots, 30$ gives the power law $N_{n} \propto n^{1.27}$. A similar power-law increase of the number of words have been already found for quasiperiodically forced systems by Pikovsky et al. (1996). To classify the complexity, we remind that for a periodic symbolic sequence the number of possible

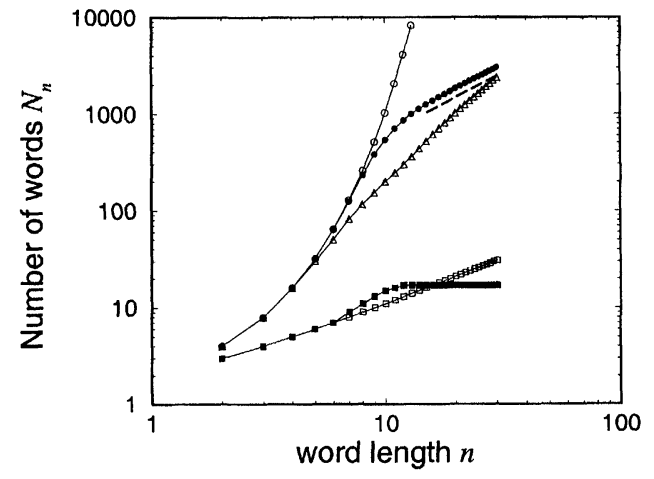

FIGURE 9 Characterization of complexity of the two-symbol symbolic sequence (filled circles). The dashed line has slope 1.27. For comparison we show the corresponding curves for the fully random sequence of two symbols (open circles), for a periodic sequence (filled boxes), and for quasiperiodic sequences with two frequencies (open boxes) and with three frequencies (open triangles). In the latter two cases the number of words grows as power laws $\propto n$ and $\propto n^{2.2}$, respectively.

words is limited by the period, thus $N_{n}$ does not grow with $n$. A quasiperiodic symbolic sequence may demonstrate the power-law increase of the number of the words. Thus, the adopted measure of complexity reveals similarity to quasiperiodically generated symbolic sequences. Remarkably, this similarity is not seen in the correlations.

\subsubsection{Correlations}

The autocorrelation function have been calculated for the funnel states $x_{i}(t)$; the results are presented in Figure 10. The correlations decay up to level $\approx$ 0.01 , but from these figures we cannot conclude what are the spectral properties of the process exactly. Indeed, according to the general spectral 

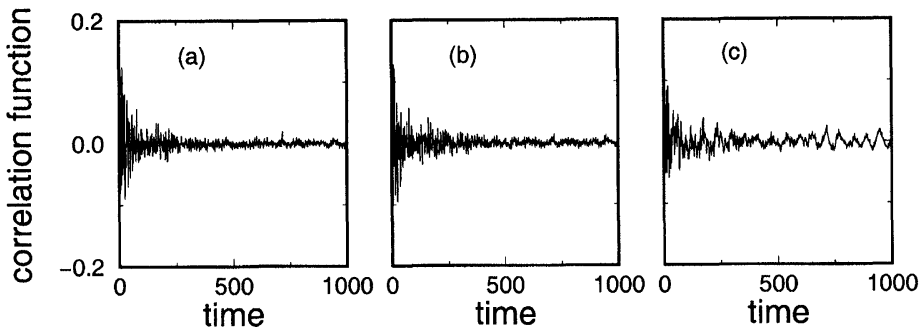

FIGURE 10 The correlation functions of time series $x_{1}(\mathrm{a}), x_{2}(\mathrm{~b})$, and $x_{3}(\mathrm{c})$. The correlations are small although we cannot conclude that they vanish for large times.
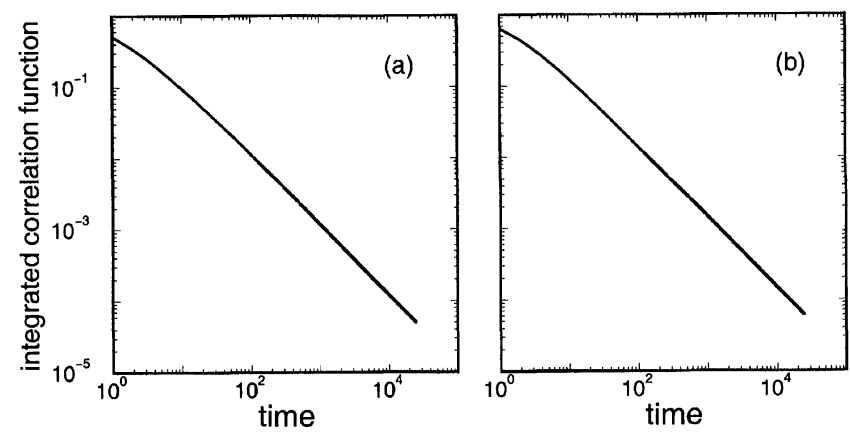

FIGURE 11 Integrated correlation functions (9): (a) the correlation function of the variable $x_{1}$ (Fig. 10a); (b): The correlations of the symbolic sequence of two symbols, as described in text.

theory (Cornfeld et al., 1982), the spectrum can have a discrete, an absolutely continuous, and a singular continuous parts. The discrete spectrum corresponds to a non-decaying part of the correlation function that oscillates periodically or quasiperiodically in time. The absolutely continuous spectrum corresponds to a decaying correlation function. Finally, the singular continuous (fractal) spectrum corresponds usually to a correlation function that decays as a power law, or to a function that occasionally has some bursts. Different examples of fractal spectra can be found in papers by Feudel et al. (1995); Pikovsky et al. (1995). To distinguish between the possibilities, we use the Ketzmerick formula (Ketzmerick et al., 1992), which gives the correlation dimension $D_{2}$ of the spectrum:

$$
c_{\mathrm{int}}(T)=\frac{1}{T} \sum_{t=0}^{T} c^{2}(t) \propto T^{-D_{2}} .
$$

Here $c(t)$ is the correlation function, and $c_{\mathrm{int}}(T)$ is the so-called integrated correlation function. We present the integrated correlation functions (for the variable $x_{1}$ and for the symbolic sequence of $1 \mathrm{~s}$ and $-1 \mathrm{~s}$ described above) in Figure 11. They show a power-law decay with $D_{2} \approx 1$. This suggests that the spectrum of the process is purely absolutely continuous. We note here, that quasiperiodic symbolic sequences demonstrate purely discrete spectrum.

\subsubsection{Stability}

As it follows from the equations of motion (4), all the trajectories are neutrally stable in the linear approximation. Correspondingly, both Lyapunov exponents are zero. However, for finite perturbations we observe instability when two neighboring trajectories fall on different sides of the borders of the triangle (6). Our numerical experiments demonstrate that preimages of the borders are 
everywhere dense. This means that any two initial points will eventually diverge, what can be described as a weak sensitivity to initial conditions. For initially closed points $\vec{x}^{(1)}, \vec{x}^{(2)}$, we can estimate the characteristic time before their images are on different sides of the cutting lines as $T_{\text {inst }} \cong|\Delta \vec{x}|^{-1}$.

\section{CONCLUSION}

We have considered a simple balanced threefunnel model of production dynamics, both for continuous and discrete order flow. In the continuous case the system demonstrates strong chaotic properties. This allows one to use the methods of chaos control to minimize the cost function. We have demonstrated that the simplest periodic orbit minimizes the switching costs, and described how to control this orbit.

The case of discrete order flow exhibits nontrivial complex dynamics. Now the dynamical properties depend on the production rates; if they are rational, the dynamics is periodic. For irrational production rates we have observed dynamics that shares properties of order and chaos. In particular, the motion is neutrally stable in the linear approximation, but has continuous spectrum. These aspects of the dynamics resemble the properties of some previously studied complex non-chaotic systems. Search for similar properties in other production systems appears to be promising, and will be subject of future research.

\section{Acknowledgements}

We thank D. Armbruster, L. Bunimovich, K. Nathansen, B. Scholz-Reiter, U. Schwarz, H.-P.
Wiendahl and M. Zaks for fruitful discussions. I.K. acknowledges support from the VWStiftung.

\section{References}

Badii, R. and Politi, A., Complexity. Hierarchical structures and scaling in physics. Cambridge University Press, Cambridge, 1997.

Bartholdi, J. J., Bunimovich, L. A. and Eisenstein, D. D. (1999) Dynamics of two- and three-worker "bucket brigade" production lines. Operations Research, 47(3), 488-491.

Bunimovich, L. A., Controlling production lines. In: Schuster, H. G. Ed., Handbook of Chaos Control, pp. 387-403, WileyVCH, Weinheim, 1999.

Chase, C., Serrano, J. and Ramadge, P. J. (1993) Periodicity and chaos from switched flow systems: contrasting examples of discretely controlled continuous systems. IEEE Trans. Automat. Control, AC-38, 70.

Cornfeld, I. P., Fomin, S. V. and Sinai, Ya. G., Ergodic Theory. Springer, New York, 1982.

Dias-Rivera, I., Armbruster, D. and Taylor, T. (1999) Periodic orbits in reentrant manufacturing systems, To be published.

Feudel, U., Pikovsky, A. S. and Zaks, M. A. (1995) Correlation properties of quasiperiodically forced two-level system. Phys. Rev. E, 51(3), $1762-1769$.

Hanson, D., Armbruster, D. and Taylor, T. (1999) On the stability of reentrant manufacturing systems, To be published.

Katok, A. and Hasselblatt, B., Introduction to the Modern Theory of Dynamical Systems. Cambridge University Press, 1995.

Ketzmerick, R., Petschel, G. and Geisel, T. (1992) Slow decay of temporal correlations in quantum systems with cantor spectra. Phys. Rev. Lett., 69, 695.

Ott, E., Grebogi, C. and Yorke, J. A. (1990) Controlling chaos. Phys. Rev. Lett., 64, 1196-1199.

Pikovsky, A., Zaks, M. and Kurths, J. (1996) Complexity of quasiperiodically driven spin system. J. Phys. A, 29, $295-302$.

Pikovsky, A. S., Zaks, M. A., Feudel, U. and Kurths, J. (1995) Singular continuous spectra in dissipative dynamics. Phys. Rev. E, 52(1), 285-296.

Schürmann, T. and Hoffmann, I. (1995) The entropy of "strange" billiards inside $n$-simplexes. J. Phys. A, 28, $5033-5039$.

Wiendahl, H.-P., Belastungsorientierte Fertigungssteuerung. Grundlagen, Verfahrensaufbau, Realisierung. Carl Hanser, München, 1987.

Zyczkowski, K. and Nishikawa, T. (1999) Linear parabolic maps on the torus. Phys. Lett. A, 259, 377-386. 


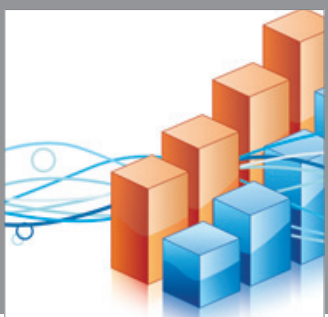

Advances in

Operations Research

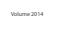

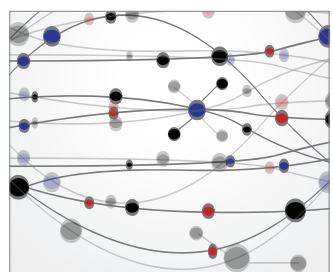

\section{The Scientific} World Journal
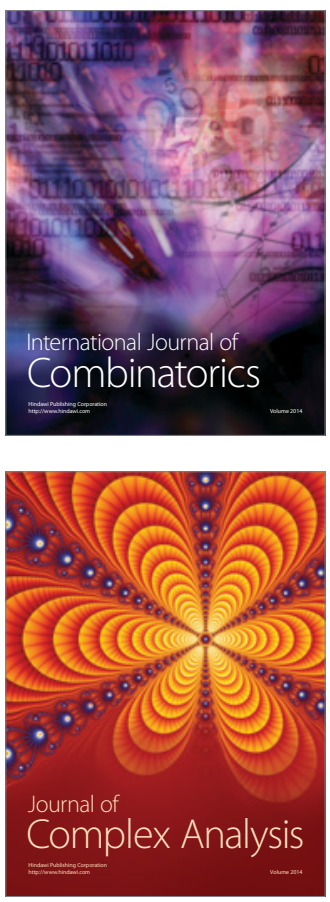

International Journal of

Mathematics and

Mathematical

Sciences
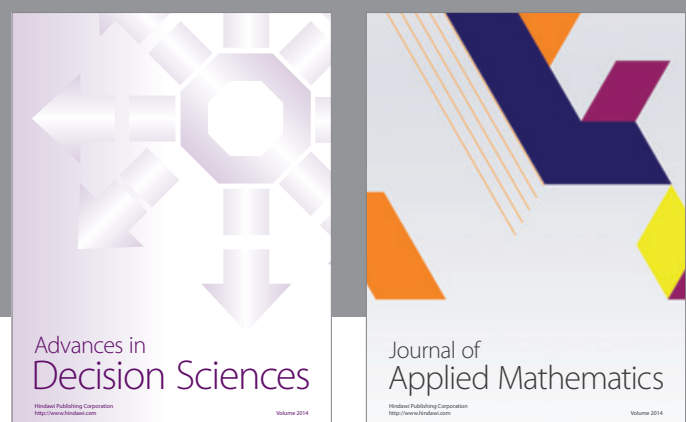

Journal of

Applied Mathematics
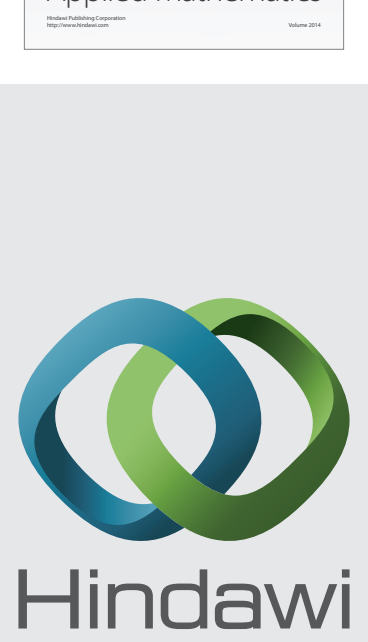

Submit your manuscripts at http://www.hindawi.com
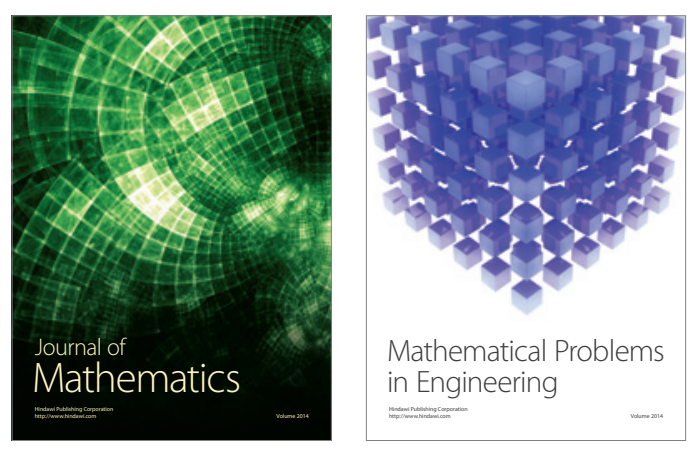

Mathematical Problems in Engineering
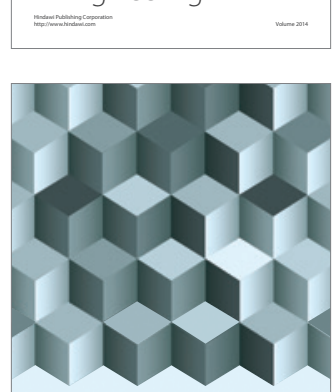

Journal of

Function Spaces
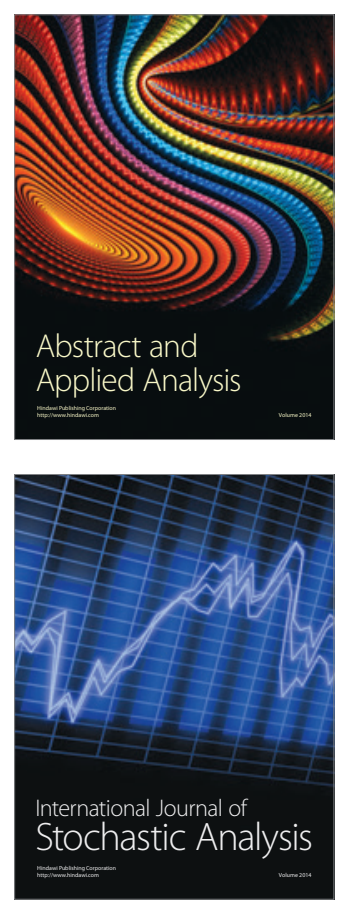

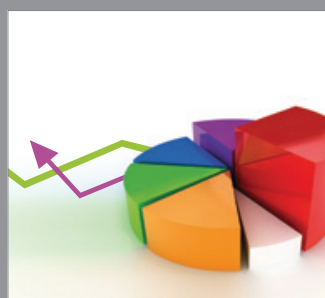

ournal of

Probability and Statistics

Promensencen
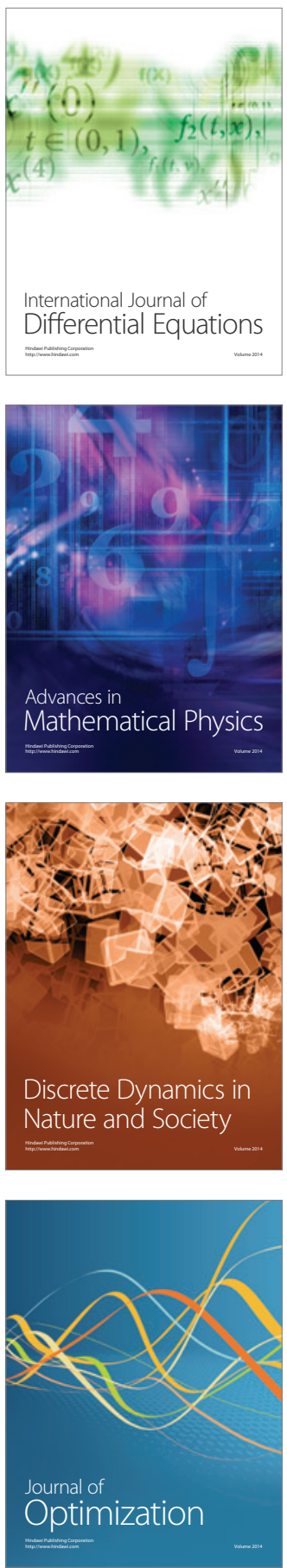